\title{
Gestión organizacional y calidad educativa en tiempos \\ de pandemia, Ate, 2021
}

\author{
Mag. Oscar Bautista-Rivas \\ bbautistari2964@ucvvirtual.edu.pe \\ firmes.777-peru@hotmail.com \\ ORCID: 0000-0001-8242-7507 \\ Universidad Cesar Vallejo \\ Línea de investigación: \\ Gestión y Calidad Educativa \\ https//ciencialatina.org/index.php/ \\ Cienciala/about/submissions\#author \\ Guidelines// \\ Cita sugerida (APA, séptima edición)
}

\section{RESUMEN}

Este trabajo de investigación estudia las principales nebulosas que enfrentan la misión organizacional y eficacia pedagógica en tiempos de pandemia. Tiene objetivos trazados de comprobar el buen manejo o mal manejo del desarrollo de las actividades institucionales. Se usó encuestas de investigación a 60 agentes educadores, entre profesores y personales administrativos de las Instituciones Educativas públicas de Ate, Perú 2021. Método se hizo un estudio hermenéutico de enfoque cualitativo de paradigma naturalista. Los primeros descubrimientos hacen referencia a la Gestión Organizacional donde los profesores y personales administrativos desenvuelven su agilidad educativa dentro del marco de clasificación donde se desenvuelve positivamente, y la eficacia formativa mejora. Resultados de validez y actividad arroja mayor porcentaje, en cuanto a la correspondencia de Gestión y la Calidad acentuando, la interrelación y la importancia de la calidad educativa. Conclusión queda demostrado la cobertura, entre la misión organizacional y la aptitud Formativa en los organismos pedagógicos de jurisdicción de Ate, Perú.

Palabras clave: gestión organizacional; calidad de la educación; eficiencia; eficacia.0 


\title{
Organizational management and educational quality in times of pandemic, Ate, 2021
}

\begin{abstract}
This research work studies the main nebulae that face the organizational mission and pedagogical effectiveness in times of pandemic. It has set objectives of verifying the good or bad management of the development of institutional activities. Research surveys were used to 60 educating agents, among teachers and administrative personnel of the public Educational Institutions of Ate, Peru 2021. Method a hermeneutical study of qualitative approach of naturalistic paradigm was made. The first discoveries refer to Organizational Management where teachers and administrative personnel develop their educational agility within the classification framework where it develops positively, and training efficiency improves. Validity and activity results show a higher percentage, regarding the correspondence of Management and Quality, emphasizing the interrelation and the importance of educational quality. Conclusion is demonstrated the coverage, between the organizational mission and the Formative aptitude in the pedagogical organisms of jurisdiction of Ate, Peru.
\end{abstract}

Keywords: organizational management; quality of education; efficiency; effectiveness.

Artículo recibido: 15 enero 2022

Aceptado para publicación: 08 febrero 2022 Correspondencia: bbautistari2964@ucvvirtual.edu.pe

Conflictos de Interés: Ninguna que declarar 


\section{INTRODUCCIÓN}

La pesquisa de este artículo profundizará la labor de la tarea organizacional y la eficiencia educativa en los centros escolares de Ate, Perú, 2021. Poseyendo en cálculo la dependencia entre dos versátiles, con el fin de demostrar un magnífico trabajo organizacional, que genere una excelente labor educativa. Pues Las invenciones constantes de un mundo globalizado según Deloitte (2016) la dinámica tecnológica organizacional, la cual, se diferencia por una fuerte competitividad y un alto conocimiento de elementos externos e internos que posibilita significativamente en la misión que desarrolla las organizaciones. Estos elementos forman que los representantes de las estructuras posean para enfrentar permutas del ambiente usando sus instrucciones de aptitud. Cantú (2016) concluyó que la eficiencia pedagógica es un problema muy grande en la educación neoliberal, presentes en el contexto social del país y en el mundo.

De esta manera, al indicar la educación de calidad correspondiente a un proyecto de logros institucionales de Educación Básica Regular (EBR). Posee la significación de trabajo que complementan todos los elementos y el círculo gubernamental, económico, social, educativo, productivo, y se analiza para llevar un esfuerzo unido con el objetivo de lograr los proyectos trazados que motivan a la respuesta a interrogantes como sigue, ¿Debido a qué punto puede ser significativa, lograr la política educativa de gestión de calidad? ¿A causa de qué componentes praxis, podría ser usado para lograr mayores significados en el desarrollo de gestión de calidad educativa? En la globalización actual en que se vive, la calidad educativa no es suficiente para construir el desarrollo de un país. Según Hellriegel (2017) el avance de la organización es un manejo exactamente concebido y de repercusión porvenir, sirve para comprender, el avance de desarrollo personal a fin de lograr la efectividad, tiene sus raíces en las ciencias de análisis funcional de conducta. Según los análisis de Palacio (2018) la gestión organizacional nace desde la importancia y capacidad de la organización para favorecer los límites de su crecimiento.

Asimismo, en la calidad educativa, las universidades están llamadas a hacer la orientación de los mecanismos de mejora al interior de sus estructuras, ya que los esquemas convencionales marcan distancia hoy en día, de las exigencias del mercado globalizado. De otra parte, concluye Narváez (2015) que la grandeza de la representación es admitir a las ordenaciones, envuelve asimismo la evaluación continua de la calidad que exige. De tal forma existen centros escolares débiles en las competencias necesarias que aplica el 
progreso consecutivo, que admitirá arrebatar disposiciones a fin de alcanzar la afectividad de las equitativas en los centros pedagógicos. Molano (2016) afirma que las organizaciones educativas, a nivel territorial y tópico, se debe observar que la gestión organizacional se encuentra en una trayectoria centrada específicamente en el aspecto administrativo y sin metas u objetivos concretos, las cuales intervengan en el logro de aprendizaje de los educandos.

Pues Los actores de la calidad, admiten la determinación organizacional de los centros pedagógicos, por agrupar los manuales de liderazgo formativo, metodologías didácticas, encargo de patrimonios. Proyectos de habilidades directivos y educativos, las privaciones de la comunicación entre los pedagógicos y estudiantes, generan efectos sobre el rendimiento escolar. Arellano (2018) afirma que esta problemática se agrava porque existe poco compromiso organizacional por parte de los magistrales, puesto que, son ellos los impulsores de los resultados educativos. Considerando que en la actualidad los lineamientos educativos son extensos y se presenta como una traba en la implementación del modelo de gestión organizacional. Si bien la demanda de instituciones educativas ha aumentado entre $27 \%$ a $47 \%$, entonces se requiere mayor gestión organizacional para mayor calidad educativa. Según Chiavenato (2014) "La administración representa la producción que se realiza con el trabajo de las personas usando recursos para así lograr los objetivos propuestos. Siendo la prioridad de los administradores eficientes y eficaces que demuestran calidad." La problemática se agudiza cuando las Instituciones Educativas no utilizan la estructura organizacional donde determine la visión, misión, objetivos y metas claras.

Es por eso, esta investigación tiene por objetivo esclarecer la misión organizacional con los principios de aptitud pedagógica en los establecimientos públicos del Perú, especialmente en el Distrito de Ate. Además para la gestión Organizacional y Calidad Educativa es un reto realizarlo su tarea, en un tiempo en que se enfrenta el COVID-19 que establece un lugar de modulación que señalará un antes y un después, por la extensión global que consiguió en corto tiempo desde que se descubrió en Wuhan, China, el 31 de diciembre del 2019, y el 11 de Marzo del 2020 ha sido puesto en conocimiento por (OMS) la potencialidad que posee las pandemias letales por su propagación y contagio en todos los países. Y esto afectó la educación global, económica, legal, laboral, medioambiental, y mayormente la salud. El contexto reciente de esta fatalidad 
epidemiológica instala de cara a diferentes desafíos. En el contorno de la gestión educativa, exige nuevas habilidades para los docentes y personales administrativos, que sean activos en usar protocolos de actuación con una perspectiva de anticipación y prevención generado por el MINSA; a fin de entrar a la nueva coyuntura de la Gestión Educacional.

Además se describe en este artículo la importancia que tiene la pesquisa y su contenido pedagógico, en las instituciones educativas, en el contexto social, mundial, nacional, local y encontradas, que se debe haber en balance que la conducta humana es un tema tan complicado y atareado; por lo general demanda investigación y elaboración de un estudio detallado al respecto, cuyo objetivo primordial es poseer un nivel de erudición de los componentes para precisar los planes de operación encaminados a optimizar los exteriores que aquejan absolutamente el desarrollo educativo. Por eso este trabajo se utilizará a manera de cotejar en forma positiva la conducta organizacional, y el desarrollo de la eficiencia escolar en el Distrito de Ate, Perú 2021. Teniendo en cuenta, que esta investigación se utilizará para dar facilidad a otras pesquisas que intenten profundizar el presente estudio; inclusive auxiliará a resolver las dificultades que se ha proporcionado en el momento de efectuar análisis de incongruente de los procesos organizacionales, y calidad educativa, con serios conflictos que superficialmente pareciera que todo estuviera normal, pero el problema se observa con un criterio de alegatos, observándose también serias dificultades entre los docentes y personales administrativos, por eso este trabajo es de reflexión que solucionará un problema histórico, por lo que faltan materiales de investigación al respecto.

\section{DESARROLLO}

Investigando en antecedentes internacionales se encontró coincidencias en referencias a las variables en investigación, Flores et al. (2017). Afirmaron la necesidad de un enfoque de la erudición organizacional, para anclarse en otros postulados de calidad educativa en Latinoamérica, enfoques complicados como el harmónico organizacional. Estos postulados conceptuales se ilustran con los resultados de una investigación positiva sobre el Conocimiento Organizacional. Además, Rueda (2017), afirma que el adelanto competitivo reside, en el ligado de prácticas originarias de enseñanza de estos ejercicios sensatos, organizadas y planeadas corregidas a adrede para el patrocinio de personajes, facultades, que benefician a la aptitud de la enseñanza. Porras (2013) en su tesis doctoral 
afirma que el trabajo de investigación produce aproximación entre el Ministerio de Educación y los centros escolares realizando un trabajo de acompañamiento sistemático, capacitado y acertado por los docentes y directivos, y los actores educativos tuvieron decisiones administrativas al respecto de la calidad educativa donde cuyos resultados fueron conocidos y discutidos para mejores incidencias. Lozada (2013), en su trabajo de investigación desarrolló la metodología descriptiva de diseño correlacional y trasversal. Llega a conclusiones donde las cualidades de liderazgo se deben practicar en los centros pedagógicos, para demostrar un ambiente de clima institucional favorable. Según Guerrero ((2012) los administradores manifiestan un concepto insuficiente para la misión de competencia de recursos humanos. Coromoto (2011) afirma que sea hecho un estudió a los manuales teóricos de la Calidad Educativa. Y concluye que la sociedad reclama la formación particular y propia del proceso educativo. En el plan de desarrollo de la educación nacional se debe tomar en cuenta las dimensiones y los factores que consideren generalizar las necesidades propias en los centros pedagógicos. Asimismo, el estudio realizado admite que las escuelas pedagógicas brinden un mejor servicio de calidad colectiva, pero a la vez instructiva. Pardo et al. (2011) plantean los objetivos que comprendan la diferencia estratégica que logre implementar las competencias administrativas científicas. Vallejo (2010), en su investigación doctoral, afirmó de los conocimientos eficientes se van modificando en el transitar de los tiempos, en los componentes de inspección eficiente, gestión eficiente total. El encargo de la aptitud total implica un cambio filosófico acerca del funcionamiento de la misión eficiente implicando la buena dirección administrativa. Valenzuela et al. (2017) afirmaron que las instituciones pedagógicas son nociones estrechamente heterogéneas en sus organizaciones, en representación individual en la enseñanza superior y media, existen la intención de las convocatorias con la cifra de los alumnos que serán utilizados para su orientación y formación curricular. García (2016) hablando de la importancia de la calidad pedagógica concluyó que se debe distinguir efectivamente los movimientos que ejecuta el director al albergar a los maestros, estudiantes y progenitores de familia al periodo de admisión, teniendo puestos los cinco sentidos, para generar un ambiente favorable a fin de resolver los problemas de forma inmediata, asimismo se debe realizar ambiente de comunicación constante. Snedy (2020) afirma que la calidad educativa y una buena organización contribuyen en este proceso de conocimiento a un nivel amplio sobre las oposiciones que 
posee al proyecto de adelanto, y ejecución para la eficiencia pedagógica que aporta la erudición de los maestros comprometidos para una buena misión organizacional.

A nivel nacional existen los trabajos previos de Ruíz (2016, donde afirma de la cultura de gestión organizacional y trabajo en equipo es importante para desarrollo equitativo de un país. Conde (2014) evalúa la importancia de la comunicación organizacional interna y consideran que es necesario uniformar las pautas y normas internas para equilibrar la descentralización y el ciclo en donde se compartirá la información interna del organismo del sector educativo. Además, Salinas (2014) propone imparcialidad en la misión didáctica, para mejoramiento administrativo eficiente. Asimismo, los materiales y recursos educacionales siempre están correlacionado con la organización ordinaria. Según Alarcón (2013) la investigación de la hipótesis se distinguió en diferentes elementos de la tarea educativa, cuyo enlace se consideró permanente en la labor de los administradores pedagógicos. Muro (2013) habla sobre un rediseño organizacional para una institución educativa; donde indica que seguir la metodología estudiada, trae un diagnóstico de doble esfuerzo y escasos ordenamientos, puesto que el compromiso reflexiona la importancia de eficaz y eficiente de la administración científica. Los autores Campos et al. (2019), coinciden que el trabajo en equipo aumenta significativamente la producción de una empresa u organización. A su vez ésta sólo se manifiesta de manera parcial o moderada y no en su totalidad. Lo cual permite ver la falta de compromiso de algunos trabajadores, por trabajar de manera conjunta para el logro de los objetivos. Finalmente, Martínez et al. (2016) bajo el método hipotético deductivo establecen que el trabajo colaborativo y la Comunicación Organizacional influye en el compromiso de los centros pedagógicos.

En el marco teórico revisado. Orozco et al. (2015). Sostienen que el progreso de gestión organizacional, tiene connotación en la práctica social y los estudios pedagógicos, y por necesidad las organizaciones se definen por sus actividades diarias, es así que por naturaleza es asimilada a partir de nacimiento hasta la muerte. Desde el pasado la familia reconocía el progreso de las organizaciones en sus roles específicos. Sin embargo, para (Brunner 2017) las organizaciones son reconocidas por su eficacia en la enseñanza, y por objetivos resulta en eficiencias pedagógicas. Según (Cabeza 2016) la gestión organizacional posee los indicadores que miden los estándares de la eficacia de las instituciones educativas, es más brindar seguridad y enseñanza a los personales 
administrativos, a los maestros y padres de familia. Es parecido a las organizaciones del siglo súper industriales, con características del cambio emergente que construye días tras día nuevos pensamientos de individuos que perciben al mundo. Los que son ajenos a esta nueva percepción del mundo permanecen en el régimen organizacional antiguo ajeno a la era súper industrial. De la misma forma es esta era de la educación globalizada juega un papel importante, la súper industrialización organizacional para la educación eficiente en el nuevo contexto social para su realización. Tejada (2009) define la gestión organizacional con sus dimensiones e indicadores para alcanzar su meta y su misión de calidad en la administración científica. Cedaro (2017) afirma la importancia que tiene la gestión estratégica, conocimiento, talento, competencias y procesos para su respectivo desarrollo de los resultados por competencia, ceñidos a la administración científica con sus dimensiones operativas.

Valdéz, (2008). Define sobre el concepto de términos de la calidad pedagógica, donde en consecuencia la sociedad adopta el proceso de formación en conjunto de valores de una cultura positivista, pero priorizando la calidad con sus dimensiones educativas. Eficiencia con sus seis indicadores y Eficacia con sus dos indicadores. Además, Nole (2016) revela que el progreso de eficacia son dignidades que van desplegando durante el transcurso de la regeneración en la institución pedagógica para la mejora de la calidad escolar, formando familiaridad, deslumbramiento en el personal administrativo, maestros, profesores y estudiantes, pero ejerciendo relevancia, eficacia, pertinencia, equidad y eficiencia. Asimismo, estas dimensiones tienen la referencia básica en las afirmaciones de la entidad globalizada UNESCO 2007, donde indica las dimensiones eficiencia, eficacia, pertinencia, relevancia y equidad. Asimismo, Lavin (2017) afirma que los maestros deben desarrollar la capacitación académica, para ser motivado a un ensanchamiento de educación eficiente, de esta manera escalando de grados desarrolle satisfactoriamente el trabajo de la educación con calidad pedagógica contextualizado. Rodríguez, (2017) dice que en la actualidad ha menguado la importancia de la calidad educativa y no es competitivo, por cuanto carece de capacitación de agentes educativos. Koontz et al. (2012). Mencionan al respecto de la dimensión eficiencia, donde consideran transformados en productos de calidad, del mismo modo mencionan sobre la dimensión que mide al mercado logrando realmente la satisfacción de sus clientes, y valora el impacto realizado al producir $100 \%$ de efectividad en el servicio de los productos de la 
calidad de súper industrial y asimismo en la calidad de la educación neoliberal. Contextualizado.

\section{METODOLOGÍA}

El tipo de esta investigación es cualitativo. Según González et al. (2017) está caracterizado hacer análisis e interpretación descriptivos en su naturaleza actual de proceso hermenéutico. Según Hernández et al. (2016), el tipo de investigación es hacer análisis específicos de características importantes del estudio sociales. En este sentido, el estudio se realizó al entorno social de los profesores y personales administrativos de las Instituciones Educativas; los cuales permitieron alcanzar el objetivo trazado. Según Hernández et al. (2016). El investigador realizará su máximo esfuerzo, a fin de prohijar el diseño que se usará durante la investigación, como forma de solución a las complicaciones esbozadas para el trabajo de investigación. Sin embargo, Hernández, Fernández \& Baptista (2006) afirma que se debe incluir todos los cuadros hermenéuticos, si vislumbra los importantes diseños conforme al requerimiento de la pesquisa con sus praxis exegéticas inductivas. La selección de estudio fue conformada intencionalmente, por el investigador, por estrictos juicios personales, y por el conocimiento que posee de la zona de pesquisa. De ahí, que la investigación quedó constituida por 60 agentes educadores, entre profesores y personales administrativos ubicado en el Distrito Ate, Perú 2021. En cuanto a la recolección de datos se aplicó la encuesta de investigación por google meet, conformado por los instrumentos y técnicas cualitativos, que presenta una escala de valoración de 4 (cuatro) alternativas, (siempre, casi siempre, casi nunca y nunca), Y se usó el programa de Excel para obtener la información de los resultados.

\section{RESULTADOS Y DISCUSIÓN}

Los datos recolectados quedaron tabulados en una matriz de doble acceso, a través de un modo manejable y enseñado en cifras necesarias y porcentuales y figuradas en gráficos de interpretación social. Que muestran las combinaciones y diferencias de las contestaciones dadas entre los profesores y personales administrativos que trabajan en los centros escolares del Distrito de Ate. La pesquisa arrojó los siguientes resultados de análisis cualitativos comprobados con varios autores de experiencias. 


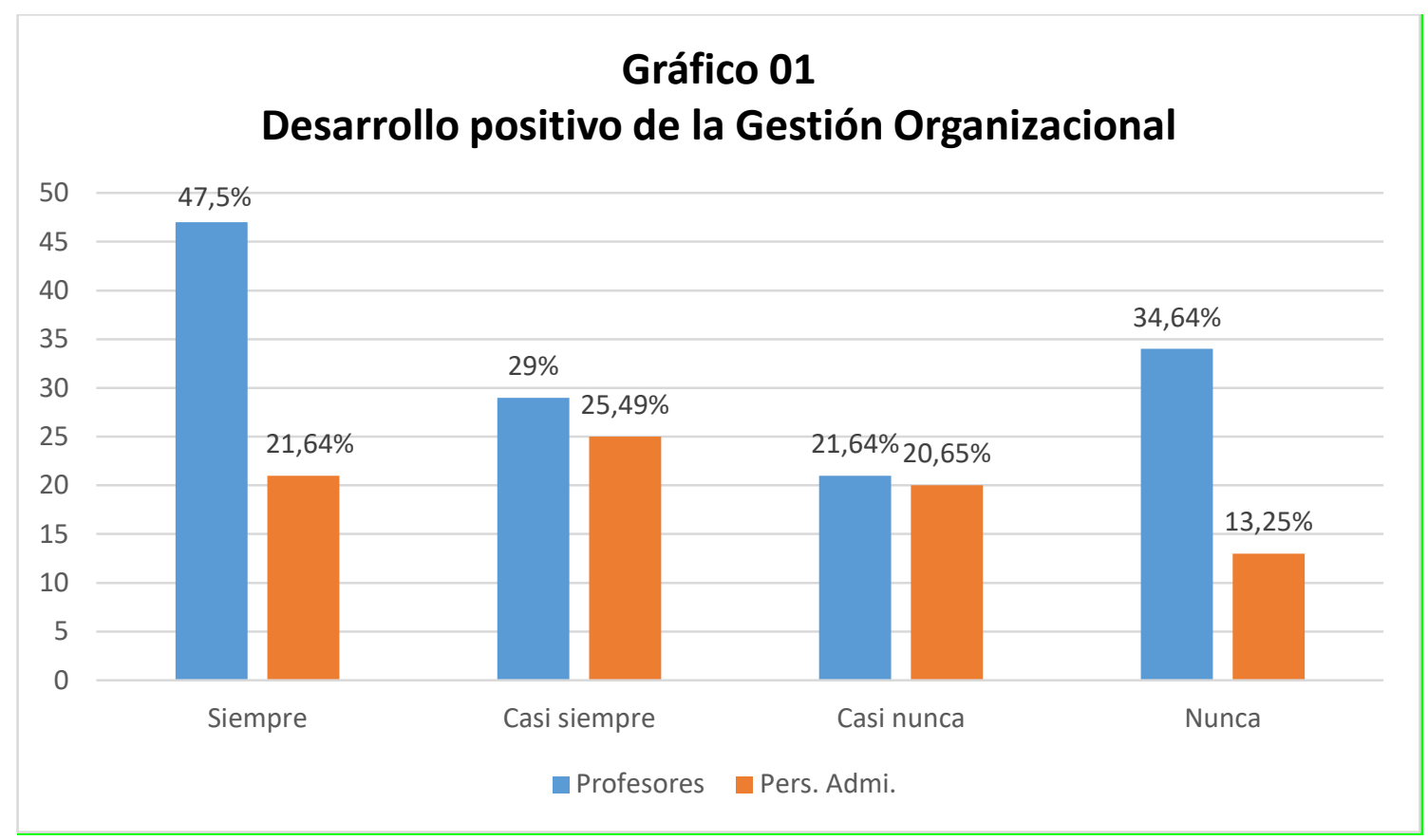

Fuente: Elaboración propia

\section{Desarrollo positivo de la Gestión Organizacional}

En esta información proporcionada, se descubre en el Gráfico 01, que los profesores atestiguan en un 47,5\% que asiduamente se revela un desarrollo positivo de la Gestión organizacional en sus instituciones educativas, que existiendo solo un 21,64\% la contestación constante por el personal administrativo. Esto refleja que para la mayoría de los agentes educadores existe un alta del proceso de desarrollo de la Gestión Organizacional Inversamente un 34,64\%. Sin embargo, para el personal administrativo que casi nunca enuncia el desarrollo de la Gestión Organizacional. Es aleatorio observar que los productos concuerdan en la réplica de casi siempre mínimos al 29\% de los encuestados. Por lo tanto, se cree que existe una buena gestión organizacional en lo concerniente a liderazgo, satisfacción, afirmación y contexto físico del centro pedagógico. Que colocan nivel de cooperación y cualidad, estableciendo su procedimiento, complacencia y paralelismo de eficiencia en la labor. Sin embargo, en algunas instituciones educativas ostentan un conocimiento despejado y consecuente, de Gestión Organizacional, y Calidad Educativa que requiere mejoramiento. En consecuencia, se producen conflictos y debilidades que se debe tomar en cuenta los personales administrativos. 


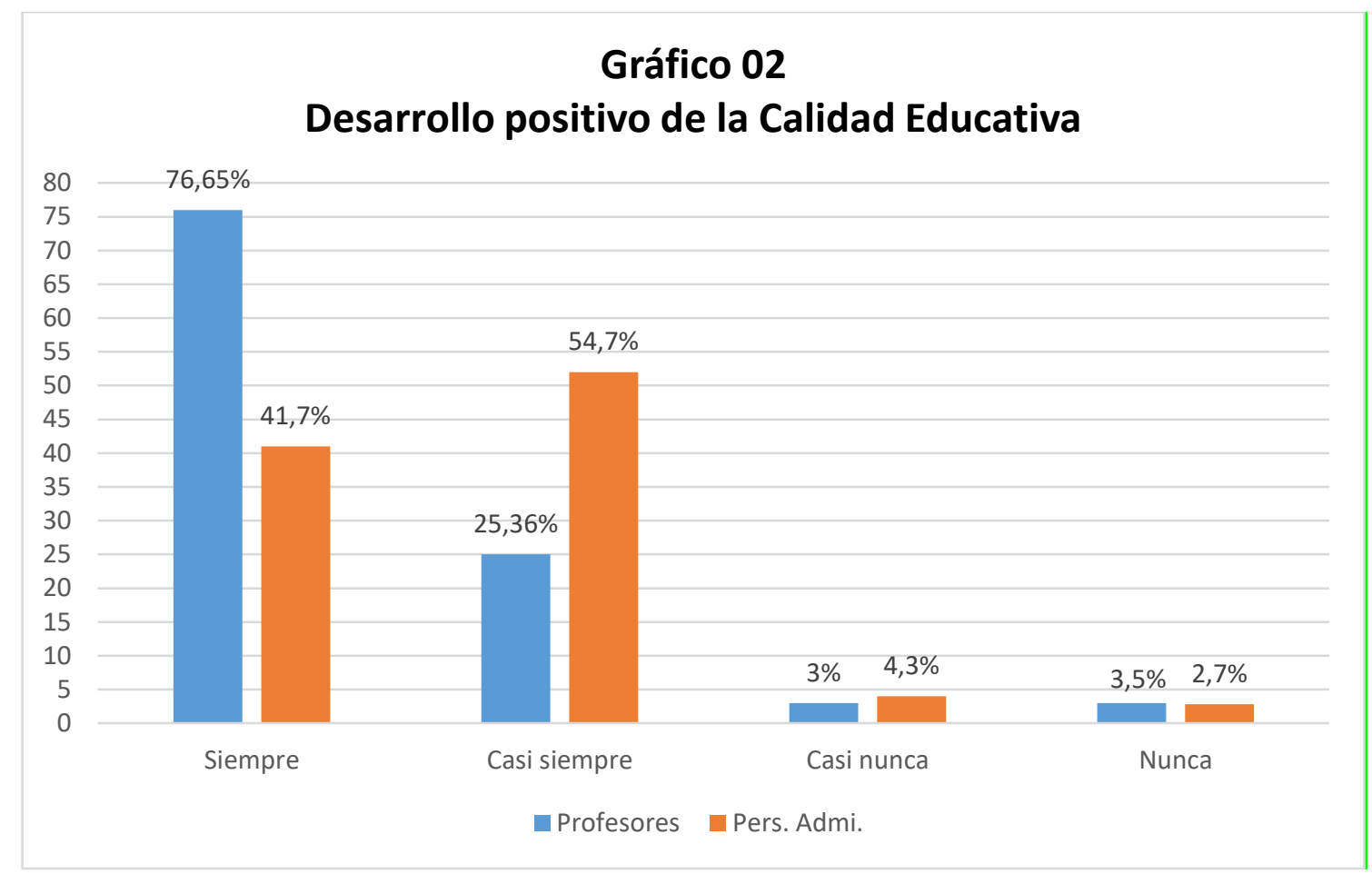

Fuente: Elaboración propia

\section{Desarrollo positivo de la Calidad Educativa}

La información conseguida en este gráfico arroja los resultados el 76,65\% de los profesores encuestados indicaron la alternativa siempre, continua de un 41,7\% como cuantía respectivo por el personal administrativo en la misma, el 54,7\% del personal administrativo cree que es eficiente la aptitud pedagógica, en los centros escolares es nivel alto, por lo tanto, quiere expresar, que se plantea una preferencia de mediana hacia alta eficacia en las instituciones de investigación, dado a las dimensiones de eficiencia y eficiencia. Al respecto Palacios (2018) señala que la calidad es producto de una buena gestión, perdurables influyendo en el comportamiento. En este marco, los servicios de una persona son admitidos como principios y premisas primordiales que mandan sus afirmaciones, cualidades y procedimientos, así pues, se considera que la organización se orienta de modo que se manifiesten con mayor eficiencia entre los miembros de la directiva institucional. 


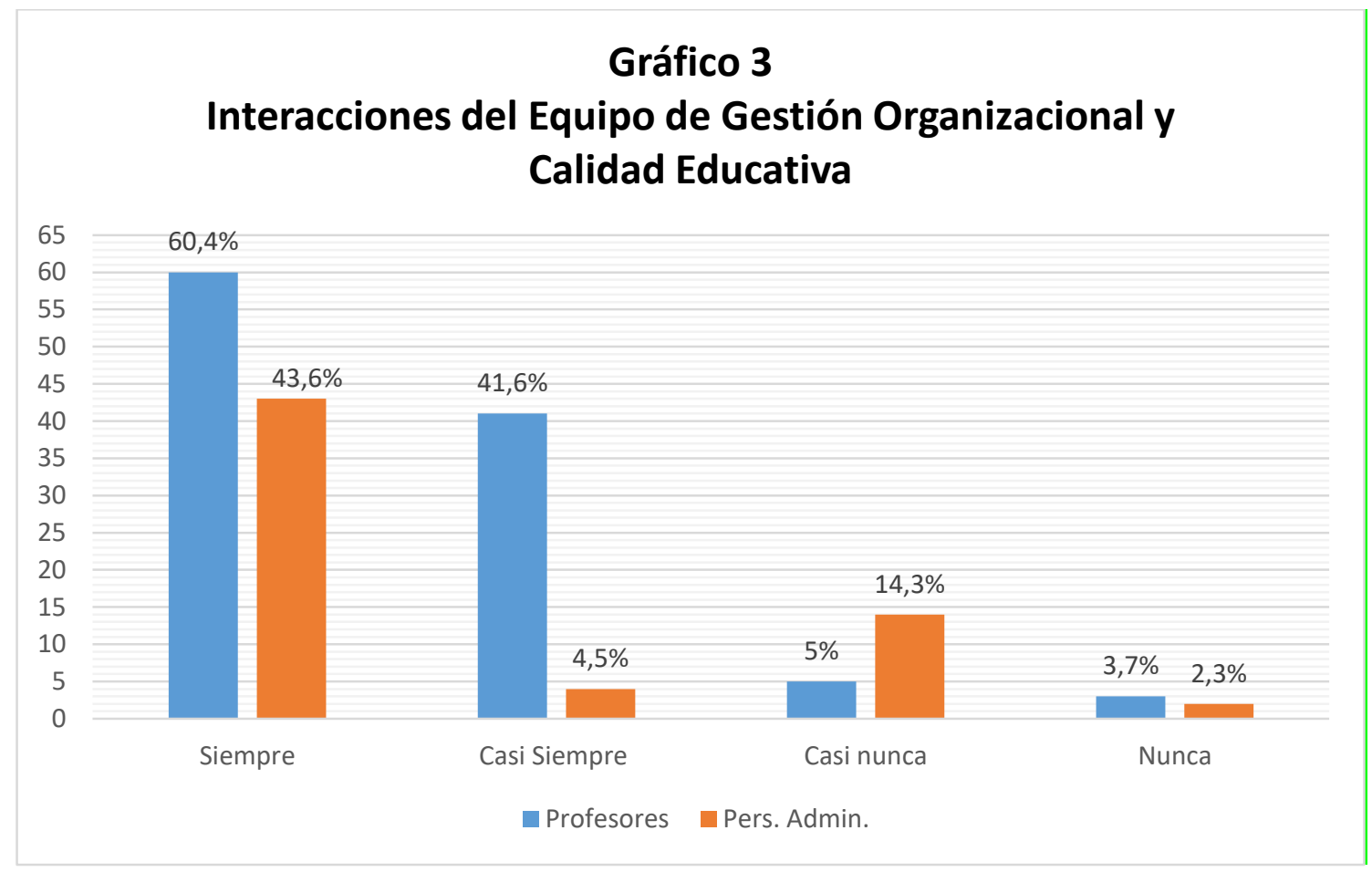

Fuente: Elaboración propia

\section{Interacciones del Equipo de Gestión Organizacional y la Calidad Educativa}

Los resultados computados para las interacciones, en el Gráfico 3, enfatizan que 60,4\% de los profesores concuerdan y siempre interactúan entre los miembros de personales administrativos de las instituciones educativas, acercándose al 43,6\% de las contestaciones obtenidas del personal administrativo. Es evidente, también, el lugar compatible entre el cálculo de las objeciones de los profesores y el personal administrativo en la respuesta casi siempre. Tal entorno irradia palpablemente, que existe una predisposición de trivial a alta declaración de los indicadores como son el trabajo en equipo, la declaración y las interacciones en equipo. 


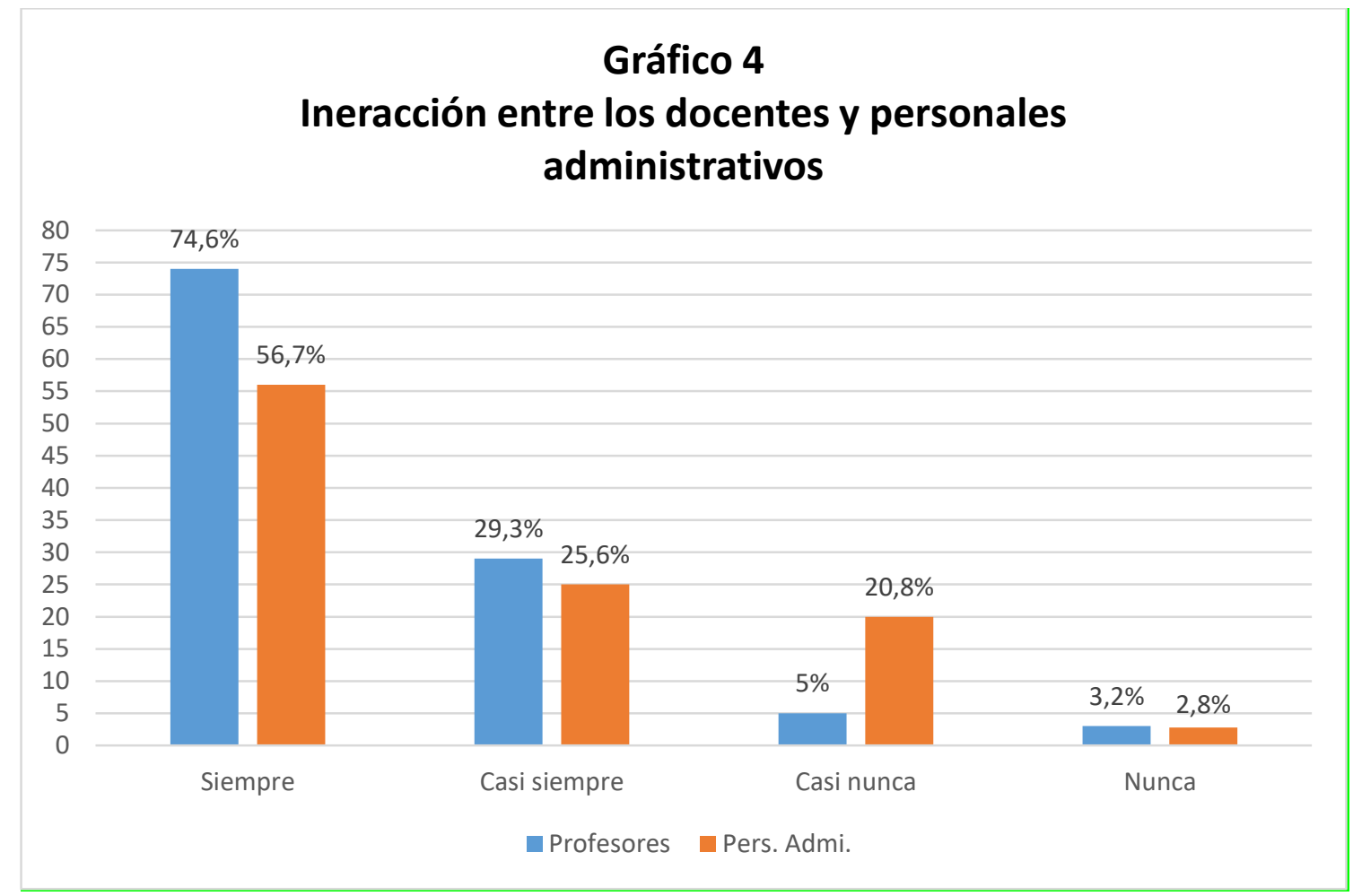

Fuente: Elaboración propia

\section{Interacción entre los docentes y Personales Administrativos}

Según Gallo Roldán et al. (2019) existen varios modelos de formación, organización e interacción entre los docentes y Personales Administrativos, de estos son los procesos sistemáticos que desarrollan estrategias y estructuras de la organización, donde hay paradigmas de eficiencias en el aspecto organizacional. Salió los resultados de interacción de calidad y rendimiento de mejoría el 74,6 \% de los profesores y el 56,7\% de personales administrativo señalan que siempre están de continua mejoría hacia la gestión organizacional y Calidad Educativa. Este resultado apunta una alta tendencia en la eficiencia pedagógica de gestión de calidad, que robustece a los centros escolares de Ate, Perú. Es positivo que una organización eficaz promueva la obtención de mejores resultados de una institución educativa, lo cual admiten la erudición organizacional para excelentes resultados de trabajo, y brindar el mejor producto tecnológico para mejorar la viabilidad y confiabilidad. Por lo tanto, requiere mejores profesionales, que sepa dirigir y organizar los recursos humanos, quien positivamente podría ser los profesores en conjunto con el personal administrativo de las instituciones educativas en Ate Perú. 


\section{CONCLUSIONES}

En conclusión, de este trabajo, los resultados señalaron la complicación del proceso en las instituciones educativas, que merecen erudiciones y más diligencia en la gestión organizacional y calidad educativa, que denotan fortalezas, oportunidades, debilidades, amenazas, (FODA) y resultados. Puesto que se han observado en las consecuencias de la investigación, con inquietud la disconformidad entre profesores y personales administrativos implicados en la investigación actual, de cómo se desenvuelve el desarrollo de la misión organizacional y la eficacia pedagógica, considerándose el liderazgo satisfactorio, afirmación y situación concreta, en donde es imperioso socavar los estudios que estén encaminados a la meditación y evidencia de los representantes para manifestar a un mejor desarrollo y ejercicio educativo. De la misma forma se reconoce en los resultados de esta investigación donde la gran mayoría de los profesores, y personales administrativos, conocen de la gestión y la eficiencia pedagógica. Sin embargo, recomiendan juntamente con $3,8 \%$ se debe mejorar en la administración científica. Asimismo, las interacciones administrativas determinan la gestión organizacional por su calidad. De esta forma aseguran el éxito en las organizaciones educativas con mayores consensos entre el Estado y el Ministerio de Educación (MINEDU) motivando expectativas, aspiraciones, enmarcados en proyectos nacionales de calidad educativa para todos.

\section{REFERENCIAS BIBLIOGRAFICAS}

Alarcón (2013). Administración educativa y calidad de la enseñanza en instituciones privadas en Lima Metropolitana. Trabajo investigativo. (USMP). Lima, Perú.

Brunner (2017). Informe de la enseñanza preeminente iberoamericana 2011. Santiago de Chile.

Chiavenato (2016). Gestión y su teoría general. 8va. versión, 3 de julio 201. Editorial Perú.

Campos (2019). Evaluación de un Establecimiento Pedagógica del territorio de Callao.

Trabajo de averiguación. (USIL), Callao.

Chiavenato (2014). Gestión de recursos humanos. McGraw Hill. Bogotá.

Cedaro (2017). Canales de comunicación y su trascendencia interna, para la administración en las Universidades Públicas y estudio de eficiencia del caso. 
Trabajo investigativo. Editorial de la Universidad Tecnológica Nacional. Concepción de Uruguay.

Coromoto (2011). Capacidad de los institutos bolivarianos en Venezuela. Averiguación científica. Universidad de León (UCL), Venezuela.

Conde (2014). Administración administrativa y la calidad educativa en las instituciones educativas públicas de la Ugel 09-Huaura 2013. Trabajo de averiguación. (UNJFSC) Huacho Perú.

Cabeza (2016). Indicadores de administración en la enseñanza preeminente como instrumento de la planeación estratégica. Estudio de coyuntura en la revista venezolana. Caracas Venezuela.

Deloitte (2016). La nueva organización y su tendencia universal con su capital humano. https://www. Deloitte. Documentos/human capital/2016.

Datum (2017). Satisfacción de Calidad y resultados en los Colegios privados. https://gestion.pe/economia/crece-preferencia-colegios-privados-caesatisfaccionsu-calidad-2181522.

Flores (2017). Modelo de administración educativa para el mejoramiento de la calidad estudiantil de la unidad educativa Mariano Suárez Veintimilla de la urbe de Ibarra. Trabajos de indagación. (UTN) Ecuador.

Guerrero (2012). Administración del ingenio humano y cultura organizacional en enseñanza elemental primaria. Trabajos de indagación. (URU) Venezuela.

Gallo Roldán (2019) La relación entre agentes educativos y familias competidores en el Centro Infantil Hermanas Misioneras de la Sociedad Cristiana: Una mirada a partir de la administración de entendimiento. Medellín Colombia.

García (2016). El logro de la calidad educativa en las instituciones públicas de enseñanza Primaria en Ensenada California Baja. Instituto de Averiguación y Desarrollo Educativo. México.

Hernández et al. (2016). Contabilidad Estratégica y el Enfoques de averiguación científica. http://estrategia.unisinu.edu.co/index.php/estrat/article/view/29

Koontz et al. (2012). Dirección una representación completa e industrial. Mc Graw Hill. México.

Muro (2013). Iniciativa de rediseño organizacional para el Instituto Cultural Peruano norteamericano-Chiclayo. Trabajo de averiguación. (USAT) Chiclayo, Perú. 
Molano (2016). Hacia la mejora de la formación profesor en la enseñanza preeminente en Colombia. Sophia. Medellín Colombia.

Nole (2016). Administración Educativa y la Optimización de la Calidad de Servicios Educativos de la escuela. (UPEU) Unidad de postgrado Ciencias Humanas y Enseñanza. Trabajos de averiguación. Ñaña, Perú.

Lozado (2013). Liderazgo y clima institucional de la Unidad Educativa María Auxiliadora de Riobamba. Trabajo de averiguación. Universidad Politécnica Salesiana, Ecuador.

Orozco et al. (2015). Táctica y entendimiento en la administración organizacional. Universidad Compañía. 13 de julio-diciembre, Bogotá Colombia.

Palacios (2018). Calidad educativa, un estudio sobre la acomodación de los sistemas de administración de la calidad empresarial a la valoración en enseñanza. https://dialnet.unirioja.es/servlet/articulo?codigo $=4183206$.

Porras (2013). Implementación del sistema de administración de la calidad en las instituciones públicas de enseñanza preescolar, elemental y media del municipio de Villavicencio. Publicación de temas diversos UNED. Trabajo de indagación científica, Colombia.

Pardo et al. (2011). La administración del ingenio humano frente al reto de las empresas competitivas. Administración Socia Recuperado de https:// revista. Lasalle. Edu.co/index./214

Ruíz (2016). Efecto de la cultura organizacional en la administración educativa de la Unidad de Educativa. Universidad Nacional de Trujillo. Trabajo de indagación. Perú.

Rodríguez (2017). Administración administrativa y calidad de servicio educativo de la Organización María Ulises Dávila Pinedo del Distrito de Morales San Martin. (UNE) Cantuta. Facultad de letras y ciencias humanas. Trabajo investigativo, Chosica Perú.

Rueda (2017). Revista Iberoamericana de Evaluación Educativa. El argumento corporativo, sumando para el aumento didáctico

Lavin, et al. (2017). Perfil y prácticas educativas del maestro orientado a la innovación en las escuelas de comercio. Revista Iberoamericana de Enseñanza Preeminente. México. 
Martínez et al. (2016). Tareas de pesquisa y la ocupación didáctica para la capacidad formativa. Lima Perú.

Tejada (2009). Los modelos recientes de administración en las empresas, administración del ingenio, y entendimiento por competencias. Psicología a partir del Caribe, Barranquilla Colombia.

Vallejo (2010). Administración de la calidad en los procesos de enseñanza-aprendizaje. Trabajo de averiguación doctoral. Madrid España.

Valenzuela et al. (2017). Creación de indicadores institucionales para la optimización de la administración y la calidad educativa. Revista Iberoamericana de Evaluación Educativa, España.

Valdés (2008). Reflexiones alrededor de la evaluación de la calidad educativa en Latinoamérica y el Caribe. Oficina Regional de Enseñanza de la UNESCO Santiago de Chile.

Snedy (2020). El mejoramiento de la administración educativa por medio de procesos de calidad debería descargar los índices de deserción en la escuela de la educación cardenal 99 Compañía de María. Trabajo de indagación doctoral. Granada Bogotá.

Salinas (2014). La calidad de la administración pedagógica y su interacción con la práctica profesor en el grado secundaria de la Organización Educativa Policía Nacional del Perú. Universidad Nacional Más grande de San Marcos. Tareas de pesquisa. Callao, Perú. 